\title{
Hamiltonian formulation, nonintegrability and local bifurcations for the Ostrovsky equation
}

\author{
Roy Choudhury, ${ }^{\dagger}$ Rossen I. Ivanov, ${ }^{\dagger 1}$ Yue Liu * \\ $\dagger$ Department of Mathematics, University of Central Florida, Orlando, FL 32816 \\ choudhur@longwood.cs.ucf.edu \\ ${ }^{\dagger}$ School of Mathematics, Trinity College Dublin, Dublin 2 Ireland \\ ivanovr@tcd.ie \\ *Department of Mathematics, University of Texas, Arlington, TX 76019 \\ yliu@uta.edu
}

\begin{abstract}
The Ostrovsky equation is a model for gravity waves propagating down a channel under the influence of Coriolis force. This equation is a modification of the famous Korteweg-de Vries equation and is also Hamiltonian. However the Ostrovsky equation is not integrable and in this contribution we prove its nonintegrability. We also study local bifurcations of its solitary waves.
\end{abstract}

MSC: 35Q35, 35Q53, 37K10

Key Words: Conservation Laws, Integrability, Local bifurcation, Ostrovsky equation.

\section{Introduction}

In this contribution we investigate the integrability and the local bifurcations of the solitary waves of the nonlinear equation

$$
\left(u_{t}-\beta u_{x x x}+2 u u_{x}\right)_{x}=\gamma u
$$

where $\beta$ and $\gamma$ are constant parameters. The equation (1.1) is known as the Ostrovsky equation [27, and is often called the Rotation-Modified Kortewegde Vries equation. It is a model for gravity waves propagating down a channel under the influence of Coriolis force. In essence, $u$ in the equation can be regarded as the fluid velocity in the $x$-direction. The physical parameter

\footnotetext{
${ }^{1}$ On leave from the Institute for Nuclear Research and Nuclear Energy, Bulgarian Academy of Sciences, Sofia, Bulgaria.
} 
$\gamma>0$ measures the effect of the Earth's rotation. The parameter $\beta$ determines the type of dispersion, namely $\beta<0$ (negative-dispersion) for surface and internal waves in the ocean and surface waves in a shallow channel with an uneven bottom. The parameter value $\beta>0$ (positive dispersion) applies to capillary waves on the surface of liquid or oblique magneto-acoustic waves in plasma. In the limit $\gamma=0$, (1.1) apparently reduces to the integrable Korteweg-de Vries equation. More details about the Ostrovsky equation can be found in [3, 8, 9, 10, 11].

The equation (1.1) has three known integrals of motion. Indeed, it can be formally rewritten in a conservation law form as follows $(\partial \equiv \partial / \partial x)$ :

$$
\begin{aligned}
u_{t} & =\left(\frac{1}{\gamma}\left(u_{t}-\beta u_{x x x}+2 u u_{x}\right)_{t}\right)_{x} \\
\left(\frac{u^{2}}{2}\right)_{t} & =\left(\beta\left(u u_{x x}-\frac{1}{2} u_{x}^{2}\right)-\frac{2}{3} u^{3}+\frac{\gamma}{2}\left(\partial^{-1} u\right)^{2}\right)_{x} \\
\left(\frac{\beta}{2} u_{x}^{2}\right. & \left.+\frac{\gamma}{2}\left(\partial^{-1} u\right)^{2}+\frac{u^{3}}{3}\right)_{t}=\left[\beta^{2}\left(u_{x} u_{x x x}-\frac{1}{2} u_{x x}^{2}\right)\right. \\
& \left.-\beta\left(2 u u_{x}^{2}-u^{2} u_{x x}\right)+\beta \gamma u_{x}\left(\partial^{-1} u\right)+\frac{\gamma^{2}}{2}\left(\partial^{-2} u\right)^{2}-\frac{u^{4}}{2}\right]_{x} .
\end{aligned}
$$

From (1.2) - (1.4) we have the following three integrals, provided the solution $u$ is in an appropriate class of functions, such that the integration (over the real line for Schwartz class functions, or over a period for periodic functions) makes sense (e.g. see [12] for details):

$$
\begin{aligned}
I[u] & =\int u \mathrm{~d} x=0, \quad \gamma \neq 0, \\
P[u] & =\frac{1}{2} \int u^{2} \mathrm{~d} x, \\
H[u] & =\int\left(\frac{\beta}{2} u_{x}^{2}+\frac{\gamma}{2}\left(\partial^{-1} u\right)^{2}+\frac{u^{3}}{3}\right) \mathrm{d} x .
\end{aligned}
$$

Now we notice that the equation (1.1) can be written as

$$
u_{t}=\frac{\partial}{\partial x} \frac{\delta H}{\delta u(x)}
$$

where the symbol $\delta / \delta u$ denotes variational derivative. Moreover, (1.8) can be further represented in a Hamiltonian form with a Hamiltonian $H$ :

$$
u_{t}=\{u, H\} .
$$

The Poisson bracket is defined as

$$
\{F, G\} \equiv \int \frac{\delta F}{\delta u(x)} \frac{\partial}{\partial x} \frac{\delta G}{\delta u(x)} \mathrm{d} x .
$$


One can check that the bracket (1.10) is anti-symmetric and satisfies Jacobi identity.

Also, we notice that the integral (1.6) has a meaning of a momentum. Indeed, it is related to the translation invariance of the Hamiltonian. Since $H[u(x+\varepsilon)]-H[u(x)] \equiv 0$, the expansion of $\int(H[u(x+\varepsilon)]-H[u(x)]) \mathrm{d} x$ in $\varepsilon$ about $\varepsilon=0$ gives (note that $u(x)=\delta P / \delta u$ )

$$
0=\int \frac{\delta H}{\delta u(x)} \frac{\partial u}{\partial x} \mathrm{~d} x=\int \frac{\delta H}{\delta u(x)} \frac{\partial}{\partial x} \frac{\delta P}{\delta u(x)} \mathrm{d} x \equiv\{H, P\}=-P_{t},
$$

i.e. $P_{t}=0$. The existence of (1.5) from the Hamiltonian viewpoint is related to the presence of the operator $\partial / \partial x$ in the Poisson brackets.

Apparently there is no second Hamiltonian formulation for the Ostrovsky equation, compatible with the presented one, i.e. this equation is not biHamiltonian. Despite the existence of the three integrals, in Section 2 we demonstrate that the equation (1.1) is not completely integrable for $\gamma \neq 0$. In Section 3 we study the local bifurcations of its solitary waves.

\section{The Integrability Test}

In our analysis we use the integrability check developed in [25, 28, 26]. (Another application of the method is presented in [18.)

This perturbative method can be briefly outlined as follows. Consider the evolution partial differential equation

$$
u_{t}=F_{1}[u]+F_{2}[u]+F_{3}[u]+\ldots
$$

where $F_{k}[u]$ is a homogeneous differential polynomial, i.e. a polynomial of the variables $u, u_{x}, u_{x x}, \ldots, \partial_{x}^{n} u$ with complex constant coefficients, satisfying the condition

$$
F_{k}[\lambda u]=\lambda^{k} F_{k}[u], \quad \lambda \in \mathbb{C} .
$$

The linear part is $F_{1}[u]=L(u)$, where $L$ is a linear differential operator of order two or higher. The representation (2.1) can be put into correspondence to a symbolic expression of the form

$$
u_{t}=u \omega\left(\xi_{1}\right)+\frac{u^{2}}{2} a_{1}\left(\xi_{1}, \xi_{2}\right)+\frac{u^{3}}{3} a_{2}\left(\xi_{1}, \xi_{2}, \xi_{3}\right)+\ldots=F
$$

where $\omega\left(\xi_{1}\right)$ is a polynomial of degree two or higher and $a_{k}\left(\xi_{1}, \xi_{2}, \ldots \xi_{k+1}\right)$ are symmetric polynomials. Each of these polynomials is related to the Fourier image of the corresponding $F_{k}[u]$ and can be obtained through a simple procedure, described e.g. in [25]. Each differential monomial $u^{n_{0}} u_{x}^{n_{1}} \ldots\left(\partial_{x}^{q} u\right)^{n_{q}}$ is represented by a symbol

$$
u^{m}\left\langle\xi_{1}^{0} \ldots \xi_{n_{0}}^{0} \xi_{n_{0}+1}^{1} \ldots \xi_{n_{0}+n_{1}}^{1} \xi_{n_{0}+n_{1}+1}^{2} \ldots \xi_{n_{0}+n_{1}+n_{2}}^{2} \ldots \xi_{m}^{q}\right\rangle
$$


where $m=n_{0}+n_{1}+\ldots+n_{q}$ and the brackets \langle\rangle denote symmetrization over all arguments $\xi_{k}$ (i.e. symmetrization with respect to the group of permutations of $m$ elements $\left.S_{m}\right)$ :

$$
\left\langle f\left(\xi_{1}, \xi_{2}, \ldots, \xi_{n}\right)\right\rangle=\frac{1}{m !} \sum_{\sigma \in S_{m}} f\left(\xi_{\sigma(1)}, \xi_{\sigma(2)}, \ldots, \xi_{\sigma(n)}\right)
$$

Also, for any function $F(2.2)$ there exists a formal recursion operator

$$
\Lambda=\eta+u \phi_{1}\left(\xi_{1}, \eta\right)+u^{2} \phi_{2}\left(\xi_{1}, \xi_{2}, \eta\right)+\ldots
$$

where the coefficients $\phi_{m}\left(\xi_{1}, \xi_{2}, \ldots \xi_{m}, \eta\right)$ can be determined recursively:

$$
\begin{gathered}
\phi_{1}\left(\xi_{1}, \eta\right)=N^{\omega}\left(\xi_{1}, \eta\right) \xi_{1} a_{1}\left(\xi_{1}, \eta\right) \\
\phi_{m}\left(\xi_{1}, \xi_{2}, \ldots \xi_{m}, \eta\right)= \\
N^{\omega}\left(\xi_{1}, \xi_{2}, \ldots \xi_{m}, \eta\right)\left\{\left(\xi_{1}+\xi_{2}+\ldots+\xi_{m}\right) a_{m}\left(\xi_{1}, \xi_{2}, \ldots \xi_{m}, \eta\right)+\right. \\
+\sum_{n=1}^{m-1}\left\langle\frac{n}{m-n+1} \phi_{n}\left(\xi_{1}, \ldots \xi_{n-1}, \xi_{n}+\ldots+\xi_{m}, \eta\right) a_{m-n}\left(\xi_{n}, \ldots \xi_{m}\right)+\right. \\
+\phi_{n}\left(\xi_{1}, \ldots \xi_{n}, \eta+\xi_{n+1}+\ldots+\xi_{m}\right) a_{m-n}\left(\xi_{n+1}, \ldots \xi_{m}, \eta\right)- \\
\left.\left.-\phi_{n}\left(\xi_{1}, \ldots \xi_{n}, \eta\right) a_{m-n}\left(\xi_{n+1}, \ldots \xi_{m}, \eta+\xi_{1}+\ldots+\xi_{n}\right)\right\rangle\right\}
\end{gathered}
$$

with

$$
N^{\omega}\left(\xi_{1}, \xi_{2}, \ldots \xi_{m}\right)=\left(\omega\left(\sum_{n=1}^{m} \xi_{n}\right)-\sum_{n=1}^{m} \omega\left(\xi_{n}\right)\right)^{-1}
$$

and the symbols \langle\rangle denote symmetrization with respect to $\xi_{1}, \xi_{2}, \ldots \xi_{m}$, (the symbol $\eta$ is not included in the symmetrization).

We need also the notion of a local function, which can be defined as follows. The function $b_{m}\left(\xi_{1}, \xi_{2}, \ldots \xi_{m}, \eta\right), m \geq 1$ is called local if all coefficients $b_{m n}\left(\xi_{1}, \xi_{2}, \ldots \xi_{m}\right), n=n_{s}, n_{s+1}, \ldots$ of its expansion as $\eta \rightarrow \infty$

$$
b_{m}\left(\xi_{1}, \xi_{2}, \ldots \xi_{m}, \eta\right)=\sum_{n=n_{s}}^{\infty} b_{m n}\left(\xi_{1}, \xi_{2}, \ldots \xi_{m}\right) \eta^{-n}
$$

are symmetric polynomials.

The integrability criterion can be summarized as follows [25]:

Theorem 1. The complete integrability of the equation (2.1), i.e. the existence of an infinite hierarchy of local symmetries or conservation laws, implies that all the coefficients (2.4) of the formal recursion operator (2.3) are local. 
The equation (1.1) can be written in the form

$$
u_{t}=\partial^{-1}\left(\beta \partial^{4}+\gamma\right) u-2 u u_{x}
$$

The symbolic representation of the operator $\partial^{-1}$ is $1 / \eta$. Moreover, Theorem 1 can be applied in this case as well [25]. The equation (2.7) can be represented in the form (2.2) with

$$
\begin{aligned}
\omega\left(\xi_{1}\right) & =\frac{\beta \xi_{1}^{4}+\gamma}{\xi_{1}} \\
a_{1}\left(\xi_{1}, \xi_{2}\right) & =-2\left(\xi_{1}+\xi_{2}\right)
\end{aligned}
$$

Then from (2.4):

$$
\begin{gathered}
\phi_{1}\left(\xi_{1}, \eta\right)=-\frac{2 \xi_{1}^{2} \eta\left(\xi_{1}+\eta\right)^{2}}{3 \beta \eta^{2} \xi_{1}^{2}\left(\xi_{1}+\eta\right)^{2}-\beta \gamma\left(\xi_{1}+\eta\right)^{2}+\gamma \eta \xi_{1}} \\
\phi_{2}\left(\xi_{1}, \xi_{2}, \eta\right)=\Phi_{2,-3}\left(\xi_{1}, \xi_{2}\right) \eta^{-3}+\Phi_{2,-4}\left(\xi_{1}, \xi_{2}\right) \eta^{-4}+\ldots
\end{gathered}
$$

The expansion of $\phi_{1}\left(\xi_{1}, \eta\right)$ (2.9a) with respect to $\eta$ is

$$
\phi_{1}\left(\xi_{1}, \eta\right)=-\frac{2}{3 \beta} \eta^{-1}-\frac{2 \gamma}{9 \beta^{2} \xi_{1}^{2}} \eta^{-3}+\frac{2 \gamma}{9 \beta^{2} \xi_{1}} \eta^{-4}+\frac{2 \gamma\left(\gamma+6 \beta \xi_{1}^{4}\right)}{27 \beta^{3} \xi_{1}^{4}} \eta^{-5}+\ldots
$$

and therefore there are obstacles to the integrability of (1.1), since the coefficients in this expansion are not polynomials in $\xi_{1}$ for $\gamma \neq 0$.

For comparison, in the integrable case $\gamma=0(\mathrm{KdV})$, we have

$$
\begin{gathered}
\phi_{1}\left(\xi_{1}, \eta\right)=-\frac{2}{3 \beta} \eta^{-1} \\
\phi_{2}\left(\xi_{1}, \xi_{2}, \eta\right)=-\frac{4}{9 \beta^{2}} \eta^{-3}+\frac{4}{9 \beta^{2}}\left(\xi_{1}+\xi_{2}\right) \eta^{-4}-\frac{4}{9 \beta^{2}}\left(\xi_{1}^{2}+\xi_{1} \xi_{1}+\xi_{2}^{2}\right) \eta^{-5}+ \\
+\frac{4}{9 \beta^{2}}\left(\xi_{1}+\xi_{2}\right)\left(\xi_{1}^{2}+\xi_{2}^{2}\right) \eta^{-6}+\ldots
\end{gathered}
$$

Therefore, the only completely integrable equation of the form (1.1) is the one with $\gamma=0$, i.e. the $\mathrm{KdV}$ equation.

\section{Solitary Waves and Local Bifurcations}

Solitary waves of the Ostrovsky equation of the form $u(x, t)=\phi(x-c t) \equiv$ $\phi(z)$ satisfy the fourth-order ODE

$$
\phi_{z z z z}-q \phi_{z z}+p \phi=-\frac{1}{\beta}\left(\phi^{2}\right)_{z z}
$$

where

$$
z \equiv x-c t, \quad p \equiv \frac{\gamma}{\beta}, \quad q \equiv-\frac{c}{\beta} .
$$


Recently, it was proved by Liu and Varlamov 22] that solitary waves $\phi$ exist if the speed $c$ satisfies that $c<2 \sqrt{\gamma \beta}, \beta>0$ with the zero mass, that is, $\int \phi \mathrm{d} z=0$. Surprisingly, even if the mass of the solitary wave of the KdV equation is not zero, it is shown [21] that the limit of the solitary waves of the Ostrovsky equation tends to the solitary wave of the $\mathrm{KdV}$ equation as the rotation parameter $\gamma$ tends to zero. For $\beta<0$, solitary waves in the form of stationary localized pulses cannot exist at all [8, 20, 31].

Equation (3.1) is invariant under the transformation $z \rightarrow-z$ and thus it is a reversible system. In this section, we use the theory of reversible systems [16, 19, 23, 24, 13, 14] to characterize the homoclinic orbits to the fixed point of (3.1), which correspond to pulses or solitary waves of the Ostrovsky equation in various regions of the $(p, q)$ plane.

The linearized system corresponding to (3.1)

$$
\phi_{z z z z}-q \phi_{z z}+p \phi=0
$$

has a fixed point

$$
\phi=\phi_{z}=\phi_{z z}=\phi_{z z z}=0 .
$$

Solutions $\phi=k e^{\lambda z}$ satisfy the characteristic equation

$$
\lambda^{4}-q \lambda^{2}+p=0,
$$

from which one may deduce that the structure of the eigenvalues is distinct in four different regions of the $(p, q)$ plane. These regions, as well as the eigenvalue structure, are shown in Fig.1. The regions are labeled as (1) (4). The boundaries of these regions are the curves marked $C_{0}$ to $C_{3}$ in Fig.1. In delineating the structure of the homoclinic orbits to fixed point (3.4) in various parts of the $(p, q)$ space, we shall first consider the bounding curves $C_{0}-C_{3}$ and their neighborhoods. Following this, we shall discuss the possible occurrence and multiplicities of homoclinic orbits to (3.4), corresponding to pulse solitary waves of the Ostrovsky equation in each of regions (1) through (4):

a. Near $C_{0}$ : This curve, on which the eigenvalues have the structure $\lambda_{1-4}=0,0, \pm \lambda, \lambda>0$ and its vicinity have been considered in the context of reversible systems in [16, 19]. In this region, a standard analysis yields the normal form on the center manifold

$$
\begin{aligned}
& \dot{X}_{1}=X_{2}, \\
& \dot{X}_{2}=\operatorname{sign}(\mu) X_{1}-\frac{3}{2} X_{1}^{2},
\end{aligned}
$$

where $\mu$ is an unfolding parameter [16]. For $\mu>0$, this yields a unique symmetric homoclinic solution

$$
X_{1}(t)=\operatorname{sech}^{2}\left(\frac{t}{2}\right)
$$


in the vicinity of $C_{0}$. One may also show persistence of this homoclinic solution in the original system (3.4) for $\mu>0$ [16].

b. Near $C_{1}$ : Near $C_{1}$, which corresponds to the eigenvalue structure $\lambda_{1-4}=0,0, \pm i \omega, \omega>0$, analysis of a four-dimensional normal from 16] shows that on the side of $C_{1}$ corresponding to Region 3 in Fig. 1 there is a $\operatorname{sech}^{2}$ homoclinic orbit. However, in Region 3, where the eigenvalue structure is that of a saddle-center $\lambda_{1-4}= \pm \lambda, \pm i \omega$, the fixed point (3.4) is non-hyperbolic. In fact it can be shown by the "antisoliton" method [8] that there are no soliton solutions of the system (3.1) for which the function $\phi(z) \rightarrow 0$ when $|z| \rightarrow \infty$ together with its derivatives.

c. Near $C_{2}$ : In this region, where $\lambda_{1-4}= \pm i \omega, \pm i \omega$, analysis of complicated normal form [4, 17] shows the possible occurrence of so-called "envelope" homoclinic solutions of the form $\operatorname{sech}(k t) e^{i \alpha \theta}$ and with oscillating tails in the so-called "subcritical" form. However, occurrence or persistence of these solutions in the full nonlinear system (3.1) is a non-trivial issue and each system must be analyzed separately [6, 15, 17.

d. Near $C_{3}$ : There is no small-amplitude bifurcation on $C_{3}$, on which $\lambda_{1-4}= \pm \lambda, \pm \lambda$ and the fixed point (3.4) remains hyperbolic. However, as we discuss below, there is a bifurcation across it causing the creation of an infinite multiplicity of homoclinic orbits.

We turn next to each of the regions 1 to 4 in Fig. 1 to discuss the possible occurrence and multiplicity of homoclinic orbits in each region.

Region 1. In this region $\lambda_{1-4}= \pm \lambda, \pm i \omega$ and the fixed point (3.4) is a saddle focus. Using a Shil'nikov type analysis, one may show 13, 14 for general reversible systems such as (3.1) that the existence of one symmetric homoclinic orbit implies the existence of an infinity of others. Hence, we expect our system (3.1) to admit an infinity of such symmetric $N$-pulses for each $N>1$. Here, a symmetric $N$-pulse oscillates $N$ times in phasespace for $z \in(-\infty, \infty)$ (or, more technically, crosses a transverse section to the primary symmetric 1 pulse $N$ times). In the context of the Ostrovsky equation, these would be $N$-peaked solitary waves, and we expect an infinite family for all $N>1$ for parameters $(p, q)$ in Region 1 of Fig.1.

Region 2. In this region $\lambda_{1-4}= \pm \lambda_{1}, \pm \lambda_{2}$ and the fixed point (3.4) is a hyperbolic saddle point. Thus, there is no a priori reason for multiplicity of homoclinic orbits in this region. However, depending on the actual form of the nonlinear term, a symmetric homoclinic orbit to (3.4) may exist (see [5, 7]). Also, depending on further conditions [1, 29], a further "orbit-flip" bifurcation may cause complex dynamics in its neighborhood. In the context of our system (3.1), these issues will need further investigation to establish possible existence of solitary wave solutions in this region of $(p, q)$ space.

Region 3. The generic situation in this region has already been considered 
in the discussion above pertaining to the region near curve $C_{1}$.

Region 4. In this region, $\lambda_{1-4}= \pm i \omega, \pm i \omega_{2}$ and (3.4) is a focus. No homoclinic orbits are known to exist in general here, although complex dynamics may occur 2, 30.

\section{Acknowledgments}

R. I. Ivanov and Y. Liu gratefully acknowledge the hospitality and support of the Mittag-Leffler Institute, Stockholm, where this research was performed during the semester program on "Wave Motion" in the Fall of 2005. R. I. Ivanov also acknowledges the funding from the Irish Research Council for Science, Engineering and Technology.

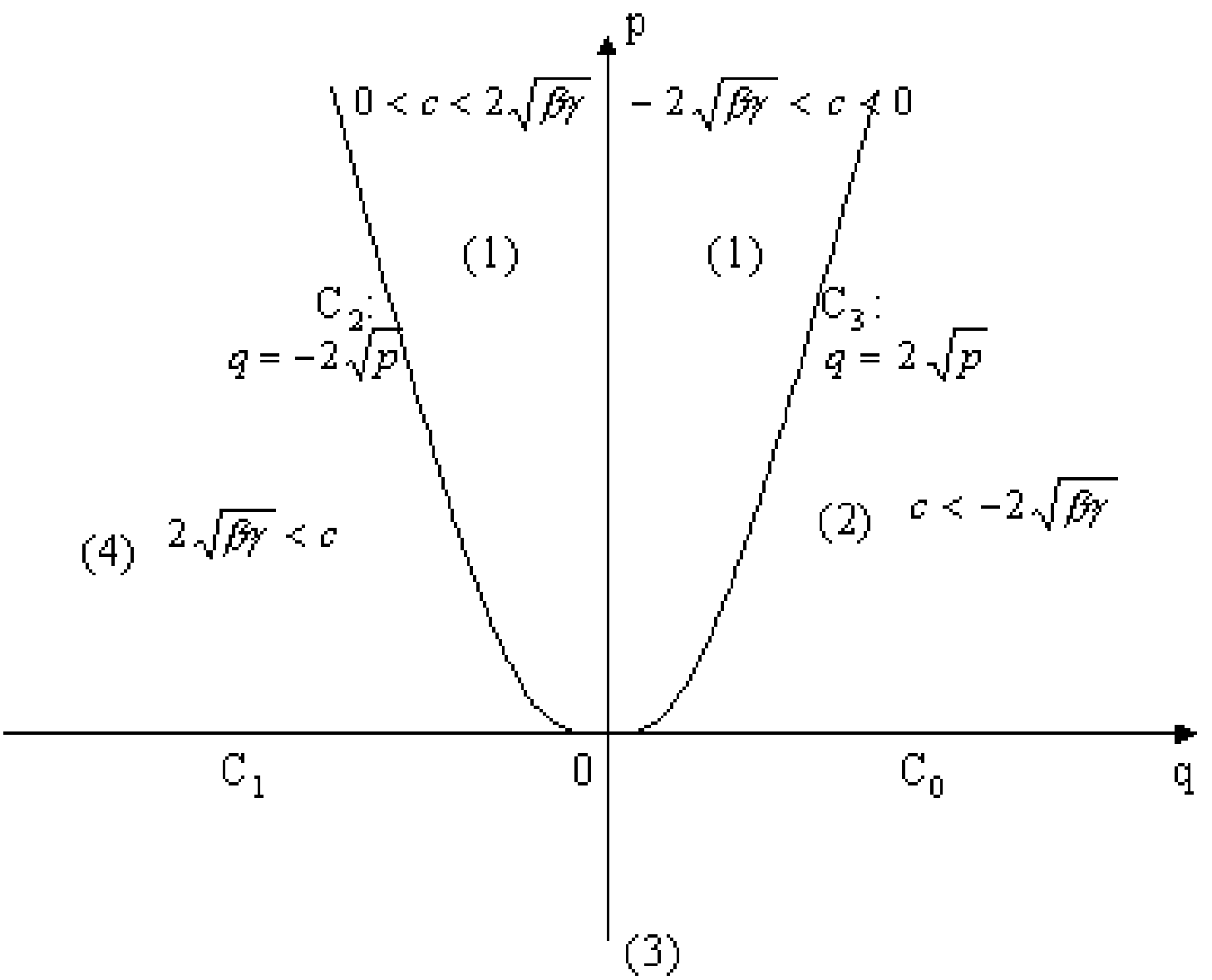

Figure 1 


\section{References}

[1] Alexander JC, Grillakis MG, Jones CKRT and Sandstede B. Stability of pulses on optical fibers with phase-sensitive amplifiers. Z Angew Math Phys 1997; 48: $175-192$.

[2] Arnol'd VI and Sevryuk MB. Oscillations and bifurcations in reversible systems. in: Nonlinear phenomena in plasma physics and hydrodynamics. Editor: Sagdeev R, Moscow: Mir, 1986.

[3] Benilov ES. On the surface waves in a shallow channel with an uneven bottom. Stud Appl Math 1992;87:1-14.

[4] Elphick C, Tirapegui E, Brachet ME, Coullet P and Iooss GA. A simple global characterization for normal forms of singular vector fields. Physica D 1988;32:488.

[5] Buffoni B, Champncys AR and Toland JF. Bifurcation and coalescence of a plethora of homoclinic orbits for a Hamiltonian system. J Dynam Diff Eq $1996 ; 8: 221-279$.

[6] Buffoni B and Groves MD. A multiplicity result for solitary gravity-capillary water waves via critical-point theory. preprint, School of Mathematical Science, University of Bath, 1996.

[7] Champneys AR and Toland JF. Bifurcation of a plethora of multi-modal homoclinic orbits for autonomous Hamiltonian systems. Nonlinearity 1993;6:665721.

[8] Galkin VN and Stepanyants YA. On the existence of stationary solitary waves in a rotating field. J Appl Math Mech 1991;55: 939-943.

[9] Gilman OA, Grimshaw R and Stepanyants YA. Approximate and numerical solutions of the stationary Ostrovsky equation. Stud Appl Math 1995; 95:115126.

[10] Grimshaw R. Evolution equations for weakly nonlinear long internal waves in a rotating fluid. Stud Appl Math 1985;73:1-33.

[11] Grimshaw R, Ostrovsky L, Shrira V and Stepanyants Y. Long nonlinear surface and internal waves in a rotating ocean. Surveys in Geophysics 1998;19:289-338.

[12] Gui G and Liu Y. Existence of the global low-regularity solutions for the Ostrovsky equation. J Funct Analysis, to appear

[13] Härterich J. Kaskaden homokliner Orbits in reversiblen dynamischen Systemen. Master's thesis, 1993

[14] Härterich J. Cascades of reversible homoclinic orbits to a saddle-focus equilibrium. Physica D 1998;112:187-200.

[15] Iooss G and Kirchgässner K. Bifurcation d'ondes Solitaires en Présence d'une Faible Tension Superficielle. C R Acad Sci Paris Sr I Math 1990;311:265-268. 
[16] Iooss G and Kirchgässner K. Water waves for small surface tension: an approach via normal form. Proc Roy Soc Edinburgh Sect A 1992;122:267-299.

[17] Iooss G and Pérouème M-c. Perturbed homoclinic solutions in reversible $1: 1$ resonance vector fields. J Diff Eq 1993;102:62-88.

[18] Ivanov RI. On the integrability of a class of nonlinear dispersive wave equations. Journal of Nonlinear Mathematical Physics 2005;12:462-468; nlin.SI/0606046

[19] Kirchgässner K. Nonlinearly resonant surface waves and homoclinic bifurcation. Advances in Applied Mechanics 1998;26:135-181.

[20] Leonov AI. The effect of earth rotation on the propagation of weak nonlinear surface and internal long oceanic waves. Ann N Y Acad Sci 1981;373:150-159.

[21] Levandosky S and Liu Y. Stability of solitary waves of the Ostrovsky equation. submitted.

[22] Liu Y and Varlamov V. Stability of solitary waves and weak rotation limit for the Ostrovsky equation. J Diff Eq 2004;203:159-183.

[23] Lombardi E. Homoclinic orbits to small periodic orbits for a class of reversible systems. Proc Roy Soc Edinburgh Sect A 1996;126:1035-54.

[24] Lombardi E. Orbits homoclinic to exponentially small periodic orbits for a class of reversible systems, Application to water waves. Arch Rational Mech Anal 1997;137:227-304.

[25] Mikhailov A and Novikov V. Perturbative symmetry approach. J Phys A 2002;35:4775-90.

[26] Olver P and Jing Ping Wang. Classification of integrable one-component systems on associative algebras. Proc London Math Soc 2000;81:566-586.

[27] Ostrovsky LA. Nonlinear internal waves in a rotating ocean. Okeanologia 1978;18:181-191.

[28] Sanders J and Jing Ping Wang. On the integrability of homogenous scalar evolution equations. J Diff Eq 1998;147:410-434.

[29] Sandstede B, Jones CKRT and Alexander JC. Existence and stability of $N$-pulses on optical fibers with phase-sensitive amplifiers. Physica D 1997;106:167-206.

[30] Sevryuk MB. Reversible systems, Lecture Notes in Mathematics, 1211, New York: Springer, 1986.

[31] Varlamov V and Liu Y. Cauchy problem for the Ostrovsky equation. Discrete Dynam Systems 2004;10:731-751. 